\title{
An Italian survey on the attitudes in treating breakthrough cancer pain in hospice
}

\author{
Sebastiano Mercadante • Patrizia Villari • \\ Alessandra Casuccio
}

Received: 1 March 2010 /Accepted: 11 May 2010 /Published online: 4 July 2010

(C) Springer-Verlag 2010

\begin{abstract}
As recognition and treatment of breakthrough cancer pain $(\mathrm{BcP})$ depend on the education and knowledge of palliative care physicians, it is important to systematically explore the attitudes of palliative care physicians in hospices or palliative care units. The aim of this study was to assess the knowledge and attitudes of hospice physicians in Italy regarding $\mathrm{BcP}$ and its treatment. All hospices existing in Italy were interviewed to gather information about provision of BP medication, drugs of choice, preferred route of administration, methods to choose the dose, and choice of BcP medication based on opioid administered for background analgesia. Of 158 hospices registered, 122 centers agreed with the interview (77.2\%). Morphine was more frequently used, either orally or parenterally. In some hospices, oral transmucosal fentanyl (OTFC) was unavailable. Most physicians provided doses of opioids proportional to the opioid basal regimen, independently of the preferred opioid or the route of administration. The choice of dose titration was equally used in patients who were prescribed OTFC or parenteral morphine. The choice of breakthrough medication on the basis of opioid basal regimen was equally distributed. These findings suggest the need for improved education on
\end{abstract}

S. Mercadante $(\triangle) \cdot$ P. Villari

Pain relief and Palliative Care Unit, La Maddalena Cancer Center,

Via San Lorenzo 312,

90146 Palermo, Italy

e-mail: terapiadeldolore@lamaddalenanet.it

S. Mercadante

Palliative Medicine Teaching, University of Palermo,

Palermo, Italy

A. Casuccio

Department of Clinical Neuroscience, University of Palermo,

Palermo, Italy behalf of physicians on the assessment and treatment of $\mathrm{BcP}$, particularly in a potentially specialized setting, such as palliative care units. The choice of $\mathrm{BcP}$ medications should be based on the best cost-efficacy ratio rather than solely on economical considerations.

Keywords Breakthrough cancer pain · Palliative care . Hospices

Breakthrough cancer pain $(\mathrm{BcP})$ is a transient exacerbation of pain that occurs either spontaneously or in relation to a trigger despite adequately controlled background pain [1]. This phenomenon is common among cancer patients, with an incidence of $40-80 \%[2,3]$. BcP is mainly managed with opioids. Traditionally, normal release opioids, known as rescue medication, are given in addition to scheduled around-the-clock analgesics. However, immediate release opioids, namely oral morphine, may take up to an hour to produce analgesia. Recently, new formulations have been developed in an effort to provide a more rapid onset of effect. Oral transmucosal fentanyl (OTFC) has been shown to be more effective than oral morphine and well tolerated [4].

Despite being a prevalent phenomenon in patients with cancer pain, $\mathrm{BcP}$ is often unrecognized and undertreated, particularly in Italy, even in a palliative care setting. In a cross-sectional multicenter study involving eight Italian oncology and palliative care centers, $\mathrm{BcP}$ was treated in $59.2 \%$ of cases with extra doses of opioids, in $19.5 \%$ with nonsteroidal anti-inflammatory drugs (NSAIDs), while $21.3 \%$ of patients had no specific treatment for BcP [5]. Thus, it seems that the crucial point for an appropriate recognition and treatment of $\mathrm{BcP}$ depends on the education and knowledge of palliative care physicians about $\mathrm{BcP}$ medication to provide the correct information and prescrip- 
tion to cancer patients and their careers. Italy represents a unique country for its specificity in cultural issues, attitudes, organization, and a low opioid consumption [6]. In the last decade in Italy, there have been investments in the field of community care and health districts under the input of new legislations, and new resources from the Health Care System have been allocated for palliative care [7]. The aim of this study was to assess the knowledge and attitudes of hospice physicians regarding $\mathrm{BcP}$ and its treatment.

\section{Methods}

The Italian registry of palliative care institutions, named Osservatorio Federazione cure palliative [1] was consulted at the official website, linked to Società Italiana di Cure Palliative to select all the palliative care units and hospices existing in Italy until September 2009. Phone interviews were planned with physicians who were delegated to provide the answers. In case of failure due to the physician's busy schedule or wrong phone number, two further attempts were made, eventually looking for information from other sources.

The following information was gathered:

- Number of unit's beds

- Provision of BcP medication

- Drugs of choice

- Preferred route of administration

- Methods to choose the dose (standard dose, proportional dose, and dose titration)

- Choice of BcP medication based on opioid administered for background analgesia

- Comments

\section{Statistical analysis}

Data were collected and analyzed by the SPSS Software 14.0 version (SPSS, Inc., Chicago, IL, USA). All continuous data are expressed as a mean \pm standard deviation of the mean. Statistical analysis of quantitative data, included descriptive statistics, was performed for all the items. Frequency analysis was performed with chi-square test.

\section{Results}

A large amount of hospices was recorded in North Italy in comparison with Southern Italy, including islands (in a rank order: Lombardia $>$ Veneto $>$ Emilia Romagna $>$ Lazio $>$ Piemonte $>$ Toscana, see Table 1). A large participation of centers contacted for the interview was obtained. Of 158 hospices registered, 122 centers agreed to the interview $(77.2 \%)$. Six centers $(3.8 \%)$ refused to participate and 30 $(19 \%)$ were unattainable or non-existent despite the publishing of their data in the website of Osservatorio Italiano di Cure Palliative. The 122 hospices had a global availability of 1,375 beds (17 day-hospital beds) with a mean of $11.2( \pm 4.5)$ beds for each hospice unit (Table 2). In Abruzzo and Valle D' Aosta, no hospice was available.

Data regarding the drug of choice for $\mathrm{BcP}$ and routes of administration are presented in Table 3 and 4, oral morphine being the most common practice. Morphine was also frequently used parenterally, subcutaneously or intravenously. In four hospices, OTFC was unavailable or bought by patients; and in one hospice, prescription of OTFC required a specific request to hospital administration (likely due to the cost of the drug).

Preferences on the doses to be used for treating $\mathrm{BcP}$ are reported in Table 5. Most physicians provided doses of opioids proportional to the opioid basal regimen (between $20 \%$ and $16 \%$ of the daily dose, in most cases), independently of the preferred opioid or the route of administration (oral morphine, subcutaneous or intravenous morphine, or OTFC). Of interest, the choice of dose titration was equally used in patients who were prescribed OTFC or parenteral morphine (intravenously or subcutaneously). Finally, the choice of breakthrough medication on the basis of opioid basal regimen was equally distributed

Table 1 Distribution of hospices and centers available for the interview (from north to south to islands)

\begin{tabular}{lrrr}
\hline & No answer & Participating centers & Total \\
\hline Lombardia & 11 & 39 & 50 \\
Veneto & 6 & 12 & 18 \\
Piemonte & 5 & 6 & 11 \\
Trentino alt & 0 & 3 & 3 \\
Friuli venez & 0 & 3 & 3 \\
Liguria & 1 & 4 & 5 \\
Emilia romag & 2 & 16 & 18 \\
Toscana & 1 & 9 & 10 \\
Umbria & 0 & 2 & 2 \\
Marche & 1 & 4 & 5 \\
Lazio & 6 & 6 & 12 \\
Molise & 0 & 1 & 1 \\
Campania & 1 & 1 & 2 \\
Puglia & 0 & 5 & 5 \\
Basilicata & 0 & 3 & 3 \\
Calabria & 0 & 2 & 2 \\
Sardegna & 0 & 3 & 3 \\
Sicilia & 2 & 3 & 5 \\
Total & 36 & 122 & \\
\hline & & & \\
\hline
\end{tabular}


Table 2 Number of hospices and mean of available beds for each unit in the different regions (from north to south islands)

\begin{tabular}{lcc}
\hline & Hospices available for interview & Mean number of available beds $( \pm \mathrm{SD})$ \\
\hline Lombardia & 39 & $11.4(3.6)$ \\
Veneto & 12 & $10.2(5.3)$ \\
Piemonte & 6 & $10.2(2.0)$ \\
Trentino alt & 3 & $8.0(3.5)$ \\
Friuli venez & 3 & $14.0(5.3)$ \\
Liguria & 4 & $10.5(1.0)$ \\
Emilia romag & 16 & $12.3(5.1)$ \\
Toscana & 9 & $8.9(2.8)$ \\
Umbria & 2 & $9.5(3.5)$ \\
Marche & 4 & $8.8(2.7)$ \\
Lazio & 6 & $19.5(7.5)$ \\
Molise & 1 & $11.0(0.0)$ \\
Campania & 1 & $10.0(0.0)$ \\
Puglia & 5 & $10.4(2.2)$ \\
Basilicata & 3 & $8.7(4.1)$ \\
Calabria & 2 & $9.5(2.1)$ \\
Sardegna & 3 & $10.0(7.0)$ \\
Sicilia & 3 & $13.3(1.1)$ \\
Total & 122 & $11.2( \pm 4.5)$ \\
\hline & &
\end{tabular}

(37 physicians answered yes, 42 physicians answered not, and others did not responded).

\section{Discussion}

In the cancer population, $\mathrm{BcP}$ is a transitory flare of pain superimposed on an otherwise stable pain pattern in patients treated with opioids [8]. Recognition and appropriate management of $\mathrm{BcP}$ has been found to enhance quality of life [9]. Although the literature has adequately addressed the biologic basis, epidemiology, and management of breakthrough pain, gaps exist in recognizing and treating $\mathrm{BcP}$ in daily practice, even in potentially specialized centers, like palliative care units.

In the last decade, there has been a growing interest in palliative care in Italy and new resources from the Health Care System have been allocated for palliative care. Active

Table 3 Drugs of choice

\begin{tabular}{lr}
\hline Morphine & 93 \\
\hline OTFC & 15 \\
Morphine/OTFC & 5 \\
Morphine/NSAIDs & 2 \\
OTFC/oxycodone & 1 \\
NSAIDs/pregabalin & 1 \\
Transdermal fentanyl & 1 \\
Total answers & $118 / 122$ \\
\hline
\end{tabular}

centers involved in palliative care progressively increased with a large distribution in Northern Italy and several hospices are now available for cancer and non cancer palliative care population [7]. In Italy, the use of opioids in the management of cancer pain has been for years at the lowest levels compared with other European countries [6], and this represents a barrier to an adequate treatment of the different types of cancer pain, including BcP. In a previous Italian survey, while the prevalence of $\mathrm{BcP}$ was $72.5 \%$, BcP was treated in $59.2 \%$ of cases with extra doses of opioids, in $19.5 \%$ with NSAIDs while $21.3 \%$ of patients had no specific treatment for $\mathrm{BcP}$ [5]. To make a comparison with a country with a consolidated tradition in the use of opioids, like UK, 81 of 87 patients in a non-specialized oncological setting had been prescribed a strong opioid as $\mathrm{BcP}$

Table 4 Routes of administrations of choice

\begin{tabular}{lr}
\hline Oral & 54 \\
\hline Subcutaneous & 23 \\
Intravenous & 14 \\
Subcutaneoua/intravenous & 6 \\
Transmucosal & 4 \\
Intramuscular & 1 \\
Oral/intravenous & 1 \\
Oral/subcutaneous & 1 \\
Subcutaneous/intramuscular & 1 \\
Total answers & $105 / 122$ \\
\hline
\end{tabular}

Some answers were incomplete 
Table 5 Frequency of titration dosing or doses proportional to background opioid dosing for breakthrough pain medication

Some answers were incomplete

medication [10], although only a minority used the opioid every time they experienced $\mathrm{BcP}$ because of individual concerns.

In this survey, the response rate was high and the majority of specialized palliative care centers provided information about their attitudes on the treatment of BcP. Interesting points emerged. For example, clear geographic differences in availability of hospice beds regrettably reflect an unequal provision and access to palliative care services across the country, with South and islands having the lowest availability of hospice beds in comparison with Northern regions, confirming differences in economical development of these areas [7]. This disparity also impeded any statistical comparison regarding $\mathrm{BcP}$ among regions.

Secondly, although BcP was treated in almost all the units, the treatment was sometime unexpected and revealed a poor knowledge of existing guidelines. Currently, the options for the management of $\mathrm{BcP}$ are relatively limited. OTFC has been shown to be effective and well tolerated in a number of clinical trials [11]. However, its use has been limited by a number of practical problems, also including issues around the cost of the product $[4,11]$. Other than obvious worse indications (such as transdermal fentanyl or pregabalin), a clear prevalence in the use of oral morphine was evidenced, despite pain usually resolves before the drug starts to work. Thus, in many instances, the decision is not based on the best knowledge, but on other reasons, as physicians' preferences, availability, and probably costs. Alternately, as patients are usually admitted to these hospices when they have a limited survival [12], they do not use OTFC because it requires active participation difficult to obtain in severely ill patients.

Finally, the modality of using $\mathrm{BcP}$ medications was explored. Controversies exist about the need for dose titration, suggested in controlled studies of OTFC [13], and the traditional method of using doses of oral morphine proportional to the daily opioid regimen $[4,14,15]$. Most physicians preferred to provide doses of opioids proportional to the basal opioid regimen, either with morphine, subcutaneously or intravenously, and OTFC. Similarly, the low number of physicians who preferred to titrate patients did so either with morphine and OTFC.
Considering the low number of respondents who were prescribed OTFC, the figures seem to confirm a tendency to use different approaches also on the basis of economical restrictions, morphine remaining the cheapest treatment for $\mathrm{BcP}$. It was confirmed by the unavailability or restrictions of OTFC in some centers. Of interest, a consistent number of hospice physicians used parenteral morphine, which should better fit timing for $\mathrm{BcP}$ in comparison with oral morphine which has an inappropriate delayed analgesic effect.

As this is the first national survey on the use of $\mathrm{BcP}$ medication, comparison with experiences of other countries is unlikely due to unavailability of data. Setting and stage of disease may influence epidemiology and treatment of BcP. Differently from the hospice setting, where prescriptions are more likely to be given and the choice is based on physicians' decision and resources, the only existing study performed in UK in still active oncologic outpatients showed that patients were determinant in decision making. Despite that almost all patients were prescribed a $\mathrm{BcP}$ medication, they did not take $\mathrm{BcP}$ medication due to lack of intensity of pain or concerns about BcP medication [10]. It has been shown in a previous study performed in homecare patients with a limited survival that the incidence of $\mathrm{BcP}$, particularly movement-related pain, may decrease with the worsening of disease, as a consequence of a reduced level of activity or because patients stop even minimal activity after feeling an increase in pain intensity [16].

As recognition and treatment of $\mathrm{BcP}$ depend on the education and knowledge of palliative care physicians, it is important to systematically explore the attitudes of palliative care physicians in hospices or palliative care units existing in Italy. The answers' rate was high, so that the sample could be quite representative of how $\mathrm{BcP}$ is treated in a country where there has been a traditional limitation in the education and use of opioids in cancer pain and economical considerations may prevail. These findings suggest the need for improved education on behalf of physicians on the assessment and treatment of $\mathrm{BcP}$, particularly in a potentially specialized setting, such as palliative care units. Data provided by this survey suggest the need of implementation of the knowledge on cancer pain and $\mathrm{BcP}$ management in all the existing palliative care programs, considering the poor availability of this information provided by schools of medicine. This topic in fact is a neglected topic during the traditional courses in the university. No specialization exists in Italy and few postgraduate courses are available. This data reflects a national modality of approach for the management of BcP. No information exists from other countries. Similar data gathered in other countries should be helpful in the recognition of an important problem as $\mathrm{BcP}$, and could suggest how to resolve them in a European perspective. The choice of $\mathrm{BcP}$ medications should be based on the best cost-efficacy ratio rather than solely on economical considerations. A range of new products with potential further 
advantages on available preparations will be available in the next future. These new preparations will be successful if health care professionals prescribe them appropriately and patients take them accordingly.

Conflict of interest No conflict of interest declared.

\section{References}

1. Osservatorio Italiano di cure palliative. www.fedcp/org

2. Caraceni A, Martini C, Zecca E, Working Group of an IASP Task Force on Cancer Pain et al (2004) Breakthrough pain characteristics and syndromes in patients with cancer pain. An international survey. Palliat Med 18:177-183

3. Mercadante S, Radbruch L, Caraceni A, Cherny N, Kaasa S, Nauck F, Ripamonti C, De Conno F, Steering Committee of the European Association for Palliative Care (EAPC) Research Network (2002) Episodic (breakthrough) pain: consensus conference of an expert working group of the European Association for Palliative Care. Cancer 94:832-839

4. Davies AN, Dickman A, Reid C, Stevens AM, Zeppetella G, Science Committee of the Association for Palliative Medicine of Great Britain and Ireland (2009) The management of cancerrelated breakthrough pain: recommendations of a task group of the Science Committee of the Association for Palliative Medicine of Great Britain and Ireland. Eur J Pain 13:331-338

5. Zecca E, Campa T, De Conno F, et al (2006) Prevalence and characteristics of breakthrough pain or severe episodic pain in cancer patients. The Italian 'Questionnaire for Severe Episodic
Pain (QIDEI) study. Abstract 170, 4th Research forum of EAPC, Venezia, 25-27 March

6. Blengini C, Joranson DE, Ryan KM (2003) Italy reforms national policy for cancer pain relief and opioids. Eur $\mathrm{J}$ Cancer Care $12: 28-34$

7. Zucco F (2007) Hospice in Italia, Ministero della Salute

8. Portenoy RK, Hagen NA (1990) Breakthrough pain: definition, prevalence, and characteristics. Pain 41:273-281

9. Slatkin NE, Rhiner MI. Improving recognition and management to enhance quality of life. http://cme.medscape.com/viewarticle/ 572129BreakthroughPain

10. Davies A, Vriens J, Kennett A, McTaggart M (2008) An observational study of oncology patients' utilization of breakthrough pain medication. J Pain Symptom Manage 35:406-411

11. Davies A (2005) Current thinking in cancer breakthrough pain management. Eur J Palliat Care 12(suppl):4-6

12. Costantini M, Beccaro M, Merlo F (2005) The last three months of life of Italian cancer patients. Methods, sample characteristics and response rate of the Italian Survey of the Diyng of cancer (ISDOC). Palliat Med 19:628-638

13. Hanks GW, De Conno F, Cherny N et al (2001) Expert working group of the research network of the European Association for Palliative Care. Morphine and alternative opioids in cancer pain: the EAPC recommendations. Br J Cancer 84:587-593

14. Zeppetella G, Ribeiro MDC (2006) Opioids for the management of breakthrough (episodic) pain in cancer patients. The Cochrane Database of Systematic Reviews doi:10.1002/14651858. CD004311. pub2. Issue 1

15. Mercadante S (2009) Breakthrough pain: on the road again. Eur J Pain 13:329-330

16. Mercadante S, Costanzo BV, Fusco F, Buttà V, Vitrano V, Casuccio A (2009) Breakthrough pain in advanced cancer patients followed at home: a longitudinal study. J Pain Symptom Manage 38:554-560 